\title{
Systematic object-invariant in-hand manipulation via reconfigurable underactuation: Introducing the RUTH gripper
}

\author{
The International Journal of \\ Robotics Research \\ 2021, Vol. 40(12-14) 1402-1418 \\ (C) The Author(s) 2021 \\ (c) (i) \\ Article reuse guidelines: \\ sagepub.com/journals-permissions \\ DOI: $10.1177 / 02783649211048929$ \\ journals.sagepub.com/home/ijr
}

@SAGE

\author{
Qiujie Lu*, Nicholas Baron*, Angus B. Clark ${ }^{*}$ and Nicolas Rojas
}

\begin{abstract}
We introduce a reconfigurable underactuated robot hand able to perform systematic prehensile in-hand manipulations regardless of object size or shape. The hand utilizes a two-degree-of-freedom five-bar linkage as the palm of the gripper, with three three-phalanx underactuated fingers, jointly controlled by a single actuator, connected to the mobile revolute joints of the palm. Three actuators are used in the robot hand system in total, one for controlling the force exerted on objects by the fingers through an underactuated tendon system, and two for changing the configuration of the palm and, thus, the positioning of the fingers. This novel layout allows decoupling grasping and manipulation, facilitating the planning and execution of in-hand manipulation operations. The reconfigurable palm provides the hand with a large grasping versatility, and allows easy computation of a map between task space and joint space for manipulation based on distance-based linkage kinematics. The motion of objects of different sizes and shapes from one pose to another is then straightforward and systematic, provided the objects are kept grasped. This is guaranteed independently and passively by the underactuated fingers using a custom tendon routing method, which allows no tendon length variation when the relative finger base positions change with palm reconfigurations. We analyze the theoretical grasping workspace and grasping and manipulation capability of the hand, present algorithms for computing the manipulation map and in-hand manipulation planning, and evaluate all these experimentally. Numerical and empirical results of several manipulation trajectories with objects of different size and shape clearly demonstrate the viability of the proposed concept.
\end{abstract}

Keywords

Underactuated robotic hand, in-hand manipulation, dexterous manipulation

\section{Introduction}

With the rising interest in robot hands as an approach to achieve task versatility in robotic systems, in-hand manipulation, in addition to robust grasping, has become an important and essential ability to improve dexterity. For robotic hands, there are two major types of grasps: power and precision grasps. The precision grasp is associated with the handling of objects between the fingertips, and is also known as a pinch grasp, whereas the power grasp involves the enveloping of the fingers around the object. Prehensile in-hand manipulation involves manipulating a grasped object by a robot hand's fingers without losing contact with it. The ability of underactuated hands to perform precision grasps and prehensile in-hand manipulation on objects is usually very limited, because the precision grasp is normally unstable and the prehensile in-hand manipulation normally requires hands with higher degrees of freedom (DOFs).

\subsection{High-DOF hands performance and control}

Several highly articulated anthropomorphic hands, with a high number of DOFs, have indeed been developed to achieve grasping and manipulation tasks (Piazza et al., 2019). For instance, the DLR hand (Grebenstein et al., 2011) has 42 motors to actuate a 2-DOF wrist and 19 DOFs in the fingers through a multiturn winder which transfers the rotational gear motion to the tendons, with a smaller number of drives necessary to achieve the same

REDS Lab, Dyson School of Design Engineering, Imperial College London, London, UK

*These authors contributed equally.

Corresponding author:

Qiujie Lu, REDS Lab, Dyson School of Design Engineering, Imperial

College London, London SW7 2DB, UK.

Email: q.lu17@imperial.ac.uk 
grasping abilities of human hands. Another example is the Gifu hand (Kawasaki et al., 2002), which has 4 motors for the thumb and 3 motors for the other fingers to achieve 16 DOFs. This hand includes a four-bar mechanism at the distal joint of each finger except the thumb design to achieve underactuation with relatively low impact on grasping abilities. In general, high-DOF robot hands are usually redundant by having actuators at each joint of the fingers, making them well suited to perform hand gestures but not necessarily reliable for prehensile in-hand manipulation as they become prone to error because of the large number of actuators. By introducing tendon-driven and joint-coupling design (Jacobsen et al., 1986; Schmitz et al., 2010; Walkler, 2004), robot hands have been undergoing continuous improvements in performance and durability to mitigate these issues.

Multiple (model-free and model-based) planning and closed-loop control frameworks have also been proposed to solve the dexterous manipulation problem for robotic grippers, that is, the process of determining how to in-hand reconfigure the grasp of an object through the coordinated motion of fingers to reach a desired position and orientation (Trinkle and Hunter, 1991; Ward-Cherrier et al., 2017). These schemes in general follow a backwards methodology, sometimes implicitly, that goes from the object to the fingers, computing the required actuator positions/torques from the sought motions of the object (Eppner et al., 2015; Okamura et al., 2000). Regarding state-of-the-art control strategies, deep reinforcement learning has been recently used to training those control frameworks for articulated anthropomorphic hands. Deep reinforcement learning has performed successfully on complex manipulation tasks with the Shadow robot hand (Shadow, 2019), but this strategy has shown to require huge amounts of feedback data (Nagabandi et al., 2020), and enormous time and energy consumption to achieve goals, with a relative low success rate and no fingertip force modulation (OpenAI et al., 2019, 2020). The Shadow hand shows capability of finger pivoting, sliding, and finger gaiting with the help of the palm and gravity, and the fingertips are not always touching the manipulated object. Indeed, performing reliable prehensile in-hand manipulation under both shape diversity and shape uncertainty with a robot hand is still an open problem (Bicchi, 2000; Billard and Kragic, 2019).

\subsection{Traditional underactuated grippers}

Underactuated robot hands, by actuating multiple finger phalanges with a single actuator through a carefully designed transmission mechanism, have fewer actuators and are simpler to control while being able to grasp diverse objects (Bai and Rojas, 2018; Hang et al., 2020; Lee et al., 2020). Multiple hand designs following this principle have been certainly designed and implemented. For example, the Velo gripper (Ciocarlie et al., 2014) can perform both parallel and fingertip grasps with a single actuator, being able to pick up small objects off a flat surface. The Ocean One hand (Stuart et al., 2017) achieves a variety of pinch and power grasps via elastic finger joints and a spring transmission. Catalano et al. (2014) proposed an adaptive synergy that allows the 19-joint hand to accommodate an arbitrary number of grasp postures using only one actuator. Teeple et al. (2020) designed a fluid-driven multi-segment soft finger for robust power and precision grasping.

Ma et al. (2013) proposed an open-source, low-cost, single-actuator 3D-printed underactuated hand with four adaptive fingers. This hand shows the capability of grasping with compliant flexure joints, following ideas previously presented in Dollar (2006). Dollar and Howe (2011) also examined a joint coupling design of underactuated grippers for unstructured environments. This work discusses how a joint coupling configuration plays an important role while grasping a wide range of object sizes and positions, showing that there is a trade-off between maximizing the successful grasp range and minimizing the contact forces during the design process. An alternative to create compliant underactuated hands is to use joints with locking mechanisms. Aukes et al. (2014) proposed a hand design which is capable of locking individual joints. Then, by locking and unlocking, the hand can adopt grasp capabilities and configurations similar to a fully actuated hand. In general, underactuated hands, by incorporating elastic and passive elements to generate self-adaptation for dealing with uncertainties (Dollar and Howe, 2010; Hirose and Umetani, 1978), have been well developed for grasping tasks. However, these characteristics and hands have been seldom leveraged for achieving dexterous manipulation while keeping control complexity low.

\subsection{Add-on designs for increasing dexterity of simple grippers}

Several robotic hands have been developed by modifying existing underactuated designs in different ways to achieve translation and rotation of objects (Bouman et al., 2020; Morino et al., 2020). The GR2 gripper (Rojas et al., 2016) is a two-fingered hand that introduces an elastic pivot joint between the fingers to enlarge the range of planar reorientation. Della Santina et al. (2018) designed an intelligent embodied tendon-driven mechanism based on turning transmission friction from a disturbance into a design tool to perform a variety of grasping and manipulation tasks. Liu et al. (2018) proposed a three-fingered gripper that is actuated by a single motor and is able to grasp objects and perform rolling manipulation with a working mode switching mechanism.

Yuan et al. (2020) attached actively driven rollers on the four-bar linkage fingertips to achieve full 6-DOF motions and continuously rotation of the grasped objects. Tincani et al. (2012) added the active surfaces to enhance the dexterity, i.e., engineered contact surfaces able to simulate different levels of friction and to apply tangential thrust to the contacted object. Indeed, by adding inflatable air cavities in soft fingertips (Lu et al., 2020), simple grippers can produce robust grasping and in-hand manipulate (soft and 
delicate) objects against gravity. Alternatively, by dynamically varying the surface material of fingers both translation and rotation can be achieved ( $\mathrm{Lu}$ et al., 2020). ChavanDafie et al. (2018) designed a pneumatic shape-shifting fingertip to enable a simple parallel jaw gripper to reorient and grasp objects by changing the contact type between the fingertips and objects. This method takes advantage of gravity to reorient the object, which makes the direction and range of rotation limited. For increasing rotation capabilities, soft fingertips can be used, when compared with rigid fingertips, they generate a larger manipulation workspace for a given gripper (Lu and Rojas, 2019). Those addon fingertip designs achieve good success on increasing the dexterity of the grippers.

Adding an extra DOF on the proximal joints of an underactuated robotic hand is also a popular method to increase hand dexterity, without increasing the actuator space excessively (Odhner et al., 2014; Righthand Robotics, 2019; Robotiq, 2016). For instance, the iHY Hand (Odhner et al., 2014) is a three-fingered underactuated hand driven by five actuators in which two of the fingers have a coupled adduction/abduction motion at the proximal joints to perform different grasps and simple reposition tasks. Similarly, the commercial ROBOTIQ gripper gives four grip types (pinch mode, wide mode, scissor mode, and basic mode) with the adduction/abduction motion at the proximal joints of two fingers separately. Another alternative is to change the morphology of the fingers to achieve a particular motion characteristic. Ma et al. (2016) proposed a curved finger design to a three-fingered underactuated hand for objects to follow a spherical surface, regardless of the object size and grasping location. However, in all these cases, the control simplicity that underactuation gives to grasping is not inherited or maintained when performing in-hand manipulation operations.

Similar to our work is the approach to enlarge the grasping capabilities of robot hands based on changing the relevant position of the proximal joints of the hand, which is equivalent to equipping it with a reconfigurable palm (Dai et al., 2009; Higashimori et al., 2005; Spiliotopoulos et al., 2018; Townsend, 2000). For instance, two of the articulated fingers of the BarrettHand (Townsend, 2000) can rotate $180^{\circ}$ around the central axis of the palm to adapt various grasping configurations. This design approach is adopted in Spiliotopoulos et al. (2018) and Higashimori et al. (2005) to achieve some particular manipulation tasks. Regarding reconfigurable palms, Dai et al. (2009) proposed the Metahand which uses a spherical mechanism as a palm, proposing later a design based on a planar linkage (Cui et al., 2018). Wei et al. (2011) analyzed the kinematics and the prototype thoroughly of the metamorphic reconfigurable palm. These works are the closest to our approach, which is also based on incorporating a reconfigurable palm in the robot hand system. However, although the hardware may seem similar, our ethos and objectives are completely different, as rather than our interest being focused on presenting the versatility of a new hand, our focus is on investigating how robot hand hardware characteristics, such as a reconfigurable palm, can be leveraged to devise simple algorithms for planning and control of in-hand manipulation operations with arbitrary objects.

\subsection{Overview}

In this article, we introduce the Reconfigurable Underactuated constant-Tendon Hand (RUTH) gripper: a three-fingered self-adaptive reconfigurable underactuated hand which decouples grasping and manipulation to achieve systematic prehensile in-hand manipulations regardless of object size or shape. The hand utilizes a twoDOF five-bar linkage as the palm of the gripper, having a total of three actuators: two for controlling the reconfiguration of the palm and one for controlling the underactuated fingers, which are connected to the mobile revolute joints of the palm. The reconfigurable palm provides the hand with large grasping versatility, while allowing the easy computation of an object-invariant map between task space and joint space for manipulation. Using this map, the inhand manipulation of objects of different sizes and shapes from one pose to another is straightforward and systematic, provided the objects are kept grasped. This step is guaranteed independently by the actuator that controls the underactuated fingers using a novel tendon routing that eliminates tendon length variations when the palm reconfigures.

In what follows, we begin by discussing the design and prototype construction of the RUTH gripper, including the tendon routing method and the electronic control system. We then analyze its grasping and manipulation characteristics, presenting algorithms for computing the mapping between object manipulation workspace and active joint space, and for computing the shortest distance in joint space to move a grasped object from its current pose to a desired one. Next, we evaluate the gripper performance on both grasping and in-hand manipulation tasks, where we examine the grasping workspace and test the grasping capability with a variety of YCB objects, and select three characteristic trajectories to analyze in-hand manipulation performance with nine standard testing samples and six daily objects. Lastly we discuss the comparison between the simulation and the gripper performance as well as future work.

\section{Design of the reconfigurable palm gripper}

The RUTH gripper shown in Figure 1 (see also Extension 1) was designed to facilitate the planning and control of prehensile in-hand manipulation by the repositioning of underactuated fingers. The developed three-fingered gripper is a completely self-contained unit, with all actuators and electronics packaged inside the gripper base, and only three actuators are needed for the co-planar in-hand manipulation. The design of the working mechanism and overall system are discussed next. 

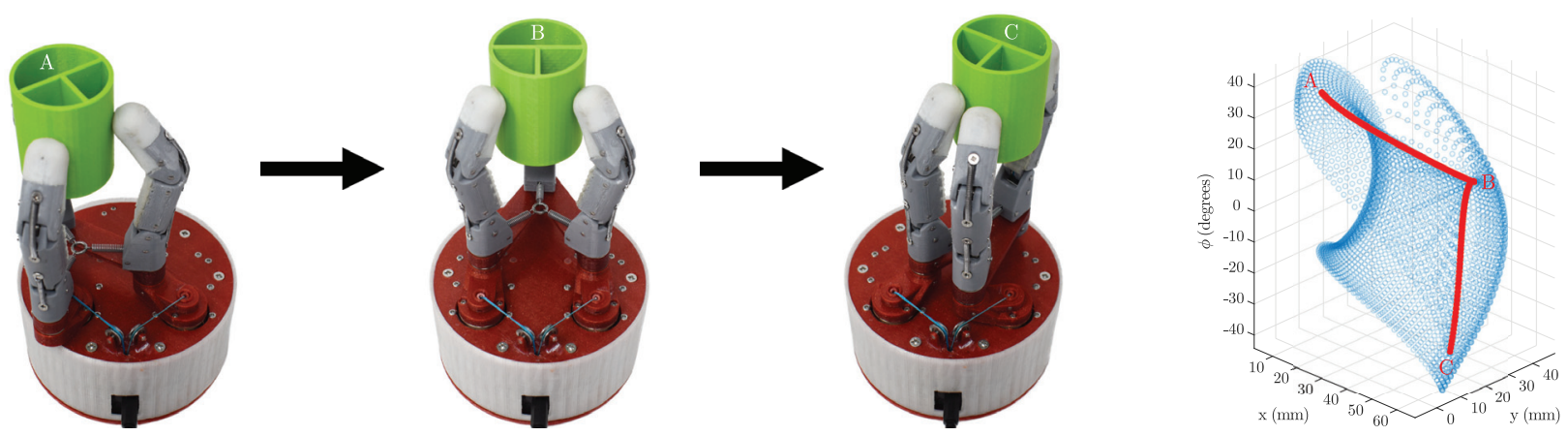

Fig. 1. The RUTH gripper decouples grasping and in-hand manipulation by exploiting palm reconfiguration and self-adaptiveness of underactuated fingers. With this gripper the motion of grasped objects of different sizes and shapes from one pose to another is straightforward and systematic, because an object-invariant map between task space and joint space can be easily pre-computed. Extension 1 clearly summarizes the article. Left: The RUTH gripper manipulating a cylindrical object from pose A to poses B and C. Right: The manipulation map with the numerical object trajectory from A to B to C.

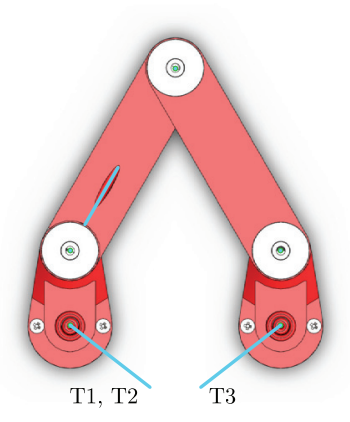

(a)

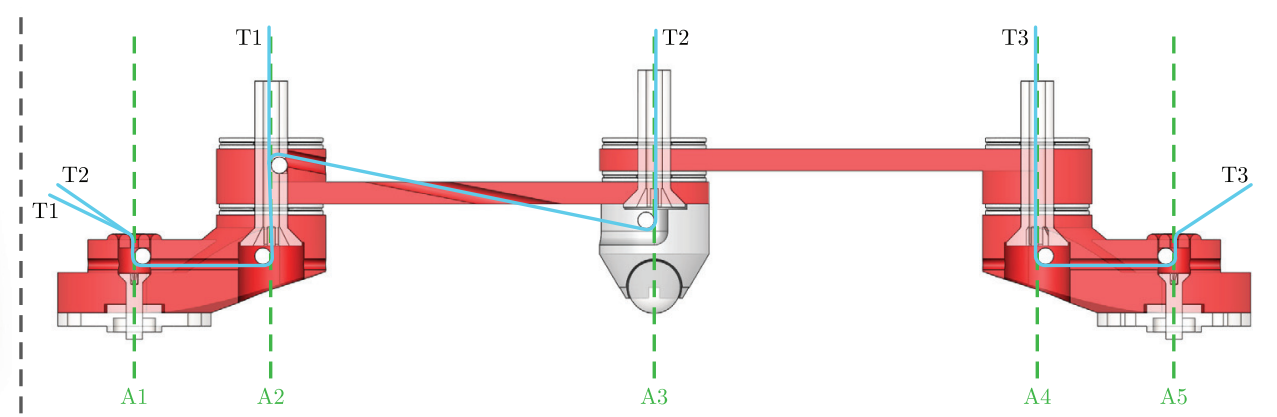

(b)

Fig. 2. CAD model showing the five-bar linkage design and configuration-independent tendon routing (blue lines, T1, T2, and T3), achieved by aligning tendon routing with the five axes of rotation (green lines, A1-A5): (a) top-view showing five-bar initial configuration and (b) unwrapped section-view showing tendon routing.

\subsection{Five-bar reconfigurable palm}

A five-bar linkage was selected for reshaping the gripper palm as it allowed three specific points, namely five-bar joints 2,3 , and 4 , to be repositioned in the $x-y$ plane through the control of only two motors, located at joints 1 and 5. When selecting dimensions for the five-bar linkage, a symmetrical structure (link $1=\operatorname{link} 4$, link $2=\operatorname{link} 3$ ) was chosen to ease manufacturing, and further to allow for the five-bar to form an equilateral triangle. This was achieved by distancing the motors axes (axes 1 and 5) at the same distance as between the five-bar joint axes 2 and $3(\operatorname{link} 2=\operatorname{link} 3=\operatorname{link} 5)$. The isolated five-bar linkage in its equilateral triangle configuration can be seen in Figure 2(a). To ensure the free-floating five-bar was supported throughout its manipulation and grasping, and ensuring minimal translation of the five-bar system in the $z$-axis (away from the top plate of the gripper), a caster wheel was placed under joint 3 . As the caster wheel required a surface to translate on, the magnitude of the five-bar was limited by the size of the gripper housing, which was limited by the size of the motors used. Through careful positioning of the three servo motors (Dynamixel MX64s), a compact housing size of $140 \mathrm{~mm} \oslash$ (by $66 \mathrm{~mm}$ tall) was developed. From this size, links 2, 3, and the motor distance (link 5) were set as $70 \mathrm{~mm}$. To provide structural rigidity the links were given cross-sectional dimensions of $20 \mathrm{~mm} \times 4 \mathrm{~mm}$ (minimum). Shorter links 1 and 4 were dimensioned such that each motor could theoretically achieve full $360^{\circ}$ motion without collisions with the other, and with a link width of $20 \mathrm{~mm}$ this gave a resulting length of $25 \mathrm{~mm}$. The links of the five-bar system were connected using bolts, and between each of the contacting faces of the links needle thrust bearings $(20 \mathrm{~mm} \oslash)$ were used to reduce the friction of the system.

To actuate the fingers of the underactuated gripper, a tendon-based method was implemented as this allowed all three fingers to be controlled using a single motor. However, one of the unique features this gripper presents is the variation in distance between the fingers and actuation motor as the five-bar linkage changes configuration. In typical underactuated grippers, the tendon exits the base of each finger and connects directly to the actuation motor. If 


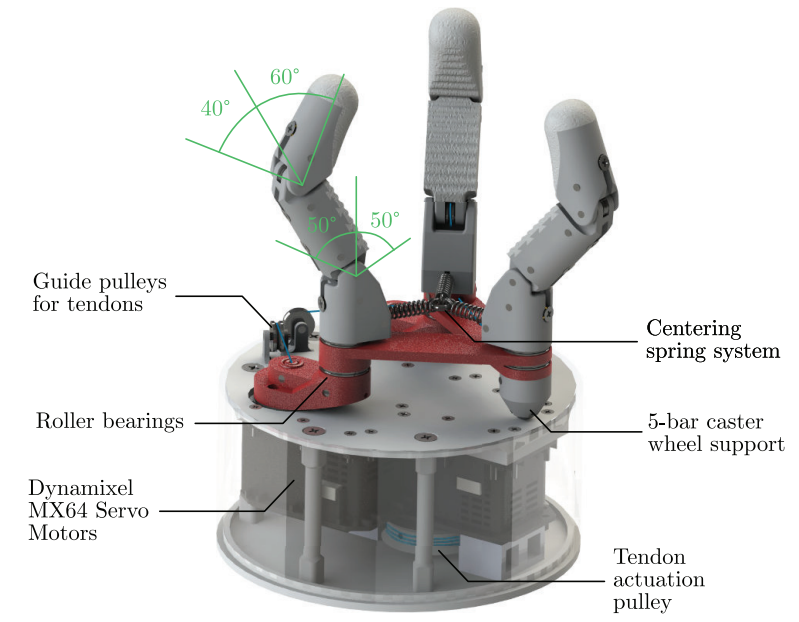

Fig. 3. CAD model showing the overall gripper structure and components. Finger joint angle limits are also shown.

this were to be implemented with the RUTH gripper, as the five-bar changes its configuration the finger tendons would vary in length relative to each other, and grasping would fail, as has been shown in previous research (Wang et al., 2020).

To overcome this issue, a constant-tendon system was implemented, where the length of each finger tendon was independent of the five-bar configuration. Each of the three tendons was passed through the five-bar mechanism, ensuring no horizontal translation occurred across the five-bar joint axes. Instead, each tendon was constrained to only vertical translation across joint axes through the use of $3 \mathrm{~mm}$ steel pins as reduced-friction guide pulleys positioned tangentially to the five-bar axes. This also presented a problem at joints 2,3, and 4, where the desired route for the tendon, along the axis, was already occupied by the bolts fixing the five-bar system together. To allow the tendons to pass along the axes, the bolts were hollowed out, and in the case of tendon routing 2 a secondary cavity was created in the side of the bolt tangential to axes 2, allowing for a steel pin to be inserted and tendon 2 to continue to its finger. On exiting the five-bar system, the tendons needed to converge to a single point (the motor), however a dual pin arrangement, as used on axes 2 for tendon 2, could not be used without restricting the movement of the five-bar system. Instead, a free-rotating ring was placed at axes 1 and 5, that allowed the tendons to converge without introducing collisions and maintaining the independent length system. A cross-section of the expanded five-bar linkage showing the tendon routing for the three individual tendons can be seen in Figure 2(b). Once the tendons exited the five-bar linkage at joints 1 and 5 , they were routed to the inverted actuation motor in the beneath housing using guide pulleys. These guide pulleys and other components of the gripper can be seen in Figure 3. The tendon routing in the base of the hand connected to the actuation motor, and the routing inside of the fingers, can be seen in Figure 4.

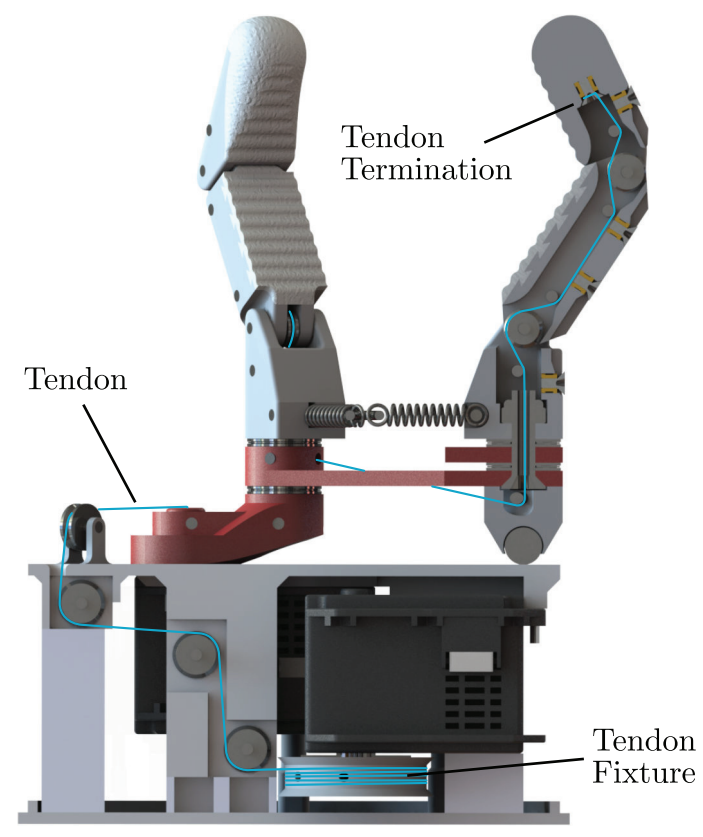

Fig. 4. Section view of the RUTH gripper, showing tendon routing in the base of the hand and in the fingers.

\subsection{Underactuated fingers}

To affix the fingers to the five-bar linkage, while also allowing rotation of the fingers, a $6 \mathrm{~mm}$ machine screw was threaded through the joint axis of each finger. The machine screw was also hollowed out to allow the tendon to pass directly through the axes, with the aforementioned no horizontal translation. The three fingers followed an identical design, with two flanges providing $\pm 50^{\circ}$ motion for the proximal flange and $+60^{\circ} /-40^{\circ}$ motion for distal flange, shown in Figure 3. Guide pulleys were placed inside the fingers at the joints to further reduce friction. To increase the grasping ability, the surfaces of the fingers were coated in textured silicone (SmoothOn Eco-Flex 0010). To return the fingers on the release of a grasp, springs were placed in channels on the back of the fingers for each joint.

To maintain the grasping capability as the five-bar configuration changes, the fingers actuation motion should be towards the center of the triangle formed by the three finger base positions (five-bar axes 2, 3, and 4). To achieve this, the orientation of each finger was controlled by a high stiffness spring attached at the base of each finger to a central ring, such that each finger is oriented towards the center of the triangle. The central ring is held in the triangle center by the three finger springs, and the ring design allows each of the finger springs to rotate around the center without experiencing the torsion expected with fixed springs. Conversely, the spring is fixed in position where it connects to the base of each finger, ensuring the spring and finger rotate towards the triangle center as one system. This spring system can be seen in Figure 3. 


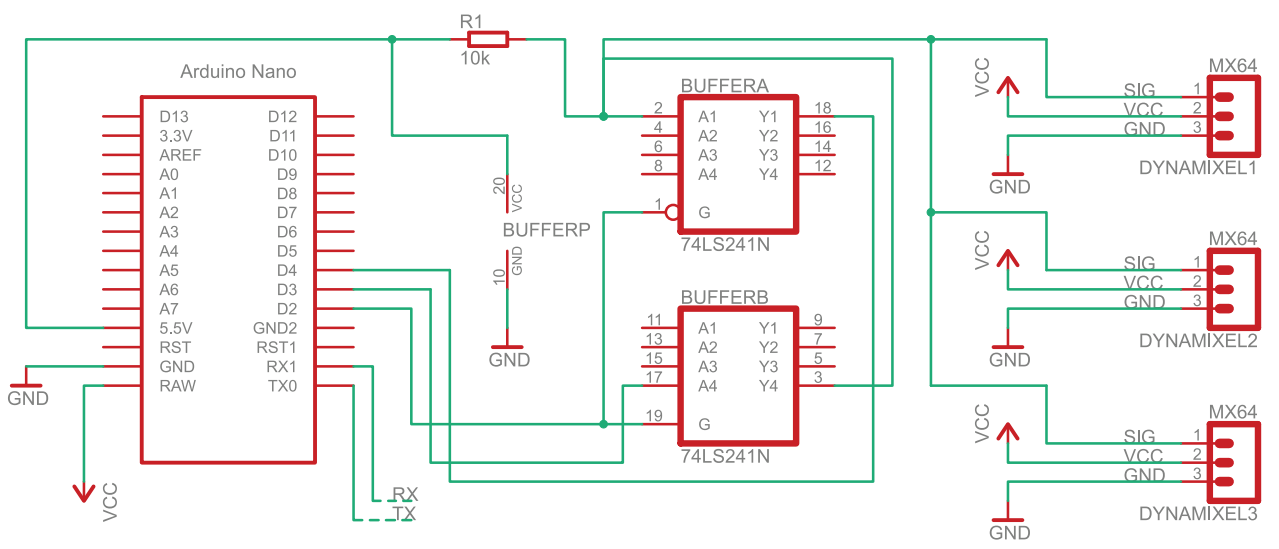

Fig. 5. Electrical schematic for controlling the RUTH gripper motors using software serial with a tristate buffer, freeing the hardware serial to enable real-time monitoring and control of the gripper.

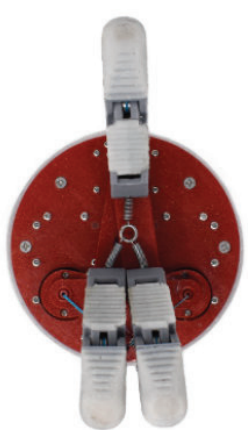

(a)

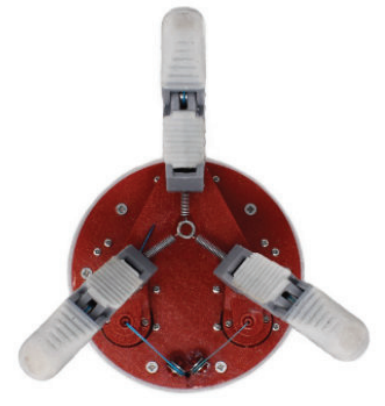

(b)

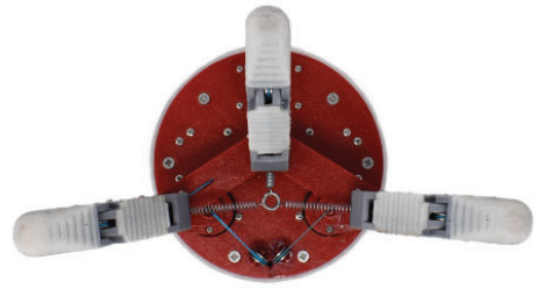

(c)

Fig. 6. Different types of grasp achievable with the RUTH gripper: (a) parallel, (b) trigonal planar, and (c) T-shape.

\subsection{Design of the prototype}

The prototype was constructed mostly from $3 \mathrm{D}$ printed parts on a single-nozzle desktop $3 \mathrm{D}$ printer. The fingers were printed out of polylactic acid (PLA), whereas the fivebar was printed out of polyethylene terephthalate glycol (PETG) for increased rigidity. This was advantageous owing to the high number of complex cavities in both. The housing for the gripper was constructed from a combination of PLA and PETG printed parts. To ensure a uniform surface on the top of the housing for the caster wheel, all surface fixtures were countersunk then filled with hot glue and smoothed till flat. An Arduino Nano microcontroller was used to control the hand using position control for the joints and torque control for the tendons, utilizing a software serial connected tristate buffer (74LS241N) to communicate using half-duplex UART protocol with the Dynamixel MX64 servo motors. This allows for real-time control of all motors, as well as providing a communications channel back from the motors to the control system (while receiving commands) and from the control system (in this case the Arduino) to a desktop computer through a hardware serial, allowing for real-time monitoring and control. This provides a significant advantage of previous proposed solutions, which typically directly connect the motors to the microcontroller. The electrical schematic can be seen in Figure 5. Thanks to the small size of the electronics they were contained within the gripper housing, with a USB socket and barrel power jack accessible on the side on the housing.

\section{Grasping and manipulation analysis of the gripper}

\subsection{Grasping configuration}

We first explore the different grasping capabilities of the RUTH gripper. Using the five-bar structure, we can reposition the fingers to allow for a variety of grasps, shown in Figure 6. In its default configuration, an equilateral triangle, the fingers form a trigonal planar grasp, ideal for power grasping spherical objects (Figure 6(b)). By rotating the motors inwards, the two short-link fingers come together to form a single "finger," forming a parallel grasp with the long-link finger (Figure 6(a)). This grasp is ideal for pinch grasping small objects and planar grasping regular cubic 
objects. Finally, by rotating the motors outwards the fivebar expands and the gripper forms a T-shape grasp, where the short-link fingers are parallel and opposite each other, with the long-link finger acting perpendicularly (Figure 6(c)). The enlarged reach of this grasp enables the grasping of larger objects, and is a combination of both the parallel and trigonal planar grasp in that it can perform power grasps on the majority of objects, with an increase in performance grasping regular cubic objects over the trigonal planar owing to the $90^{\circ}$ rotated fingers, rather than $120^{\circ}$.

\subsection{Feasible grasping workspace}

The feasible grasping workspace of the gripper is the set of positions in which an object can lie relative to the base of the hand, the palm, and be successfully grasped. It is possible to achieve a range of different grasping positions as the five-bar linkage can be reconfigured such that the center point of the proximal joints of the fingers is moved underneath the position of the center of the object. The twodimensional grasping workspace is therefore given by the set of positions that the center point of the proximal joints of the fingers, namely $\mathbf{P}_{2}, \mathbf{P}_{3}$, and $\mathbf{P}_{4}$, can achieve.

The positions of the palm's base joints, namely $\mathbf{P}_{1}$ and $\mathbf{P}_{5}$, are known and the positions of joints $\mathbf{P}_{2}$ and $\mathbf{P}_{4}$ are determined by the input angles of the actuators, say $\theta_{1}$ and $\theta_{2}$, such that

$$
\begin{aligned}
& \mathbf{P}_{2}=\mathbf{P}_{1}+l_{1}\left[\cos \theta_{1} \sin \theta_{1}\right]^{\mathrm{T}} \\
& \mathbf{P}_{4}=\mathbf{P}_{5}+l_{1}\left[\cos \theta_{2} \sin \theta_{2}\right]^{\mathrm{T}}
\end{aligned}
$$

The position of $\mathbf{P}_{3}$ can then be obtained using bilateration (Baron et al., 2019; Rojas, 2012) as

$$
\mathbf{P}_{3}=\mathbf{P}_{2}+\mathbf{Z}_{2,4,3}\left(\mathbf{P}_{4}-\mathbf{P}_{2}\right)
$$

where

$$
\mathbf{Z}_{2,4,3}=\frac{1}{2 d_{2,4}^{2}}\left[\begin{array}{cc}
d_{2,4}^{2} & -4 A_{2,4,3} \\
4 A_{2,4,3} & d_{2,4}^{2}
\end{array}\right]
$$

with $A_{2,4,3}=\frac{1}{4} \sqrt{\left(d_{2,4}^{2}+2 l_{2}^{2}\right)^{2}-2\left(d_{2,4}^{4}+2 l_{2}^{4}\right)}$ and $d_{i, j}$ being the distance between $\mathbf{P}_{i}$ and $\mathbf{P}_{j}$. The sign of $A_{2,4,3}$ determines whether $\mathbf{P}_{3}$ lies to the left or the right of the vector from $\mathbf{P}_{2}$ to $\mathbf{P}_{4}$; herein, the sign of $A_{2,4,3}$ is positive as it is desired that $\mathbf{P}_{3}$ lies always to the left.

The center point, $\mathbf{C}$, of the proximal joints of the fingers is then given by

$$
\mathbf{C}=\frac{\mathbf{P}_{2}+\mathbf{P}_{3}+\mathbf{P}_{4}}{3}
$$

The numerical grasping workspace of the gripper can be obtained by sweeping through the possible input actuator angles, $\theta_{1}$ and $\theta_{2}$, and computing the set of positions of $\mathbf{C}$ using (1)-(4). The only mechanical constraint that needs to be taken into account is the links cannot collide with the

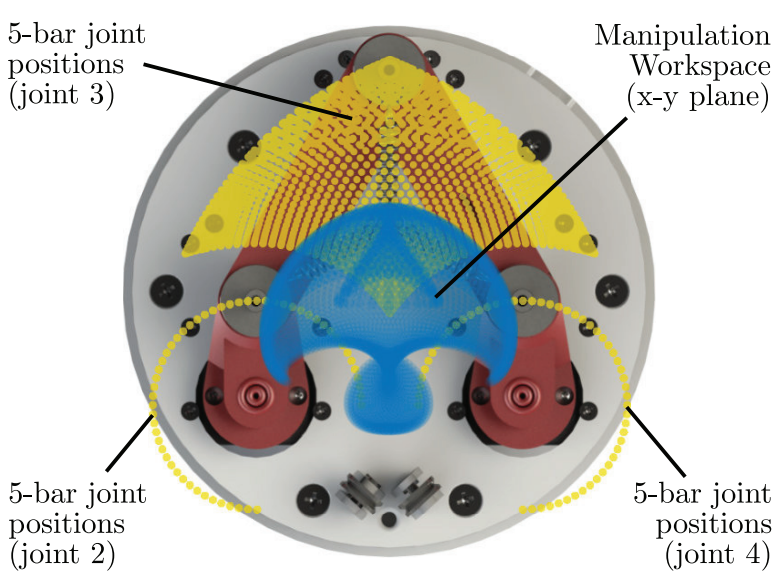

Fig. 7. Manipulation in $x-y$ in the workspace of the RUTH gripper (blue) with respect to the five-bar mechanism and gripper housing (fingers removed for clarity). Five-bar joint positions for joints 2, 3, and 4 are also shown (yellow).

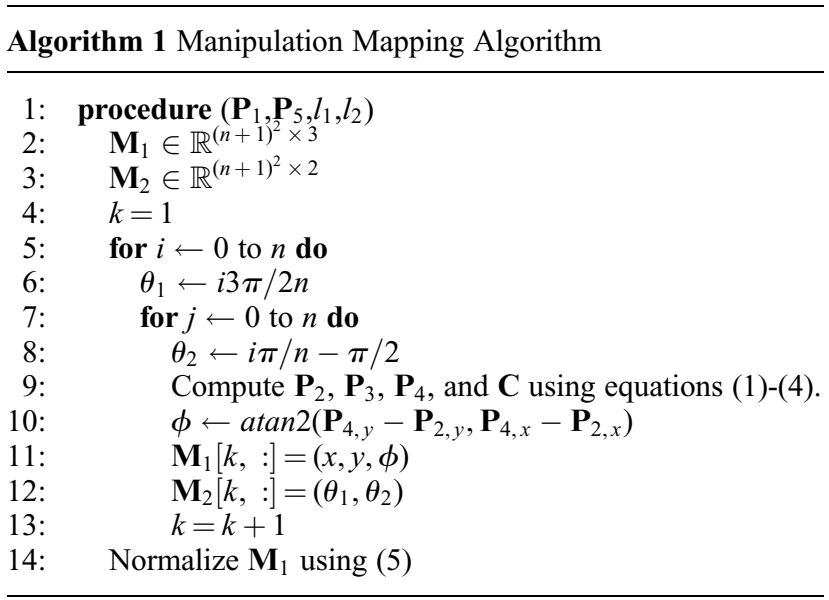

tendons passing into the base joints $\mathbf{P}_{1}$ and $\mathbf{P}_{5}$. Following this, if $\theta_{1}$ and $\theta_{2}$ are defined as the angles taken anticlockwise from the $x$-axis to the vectors from $\mathbf{P}_{1}$ to $\mathbf{P}_{2}$, and $\mathbf{P}_{5}$ to $\mathbf{P}_{4}$, respectively, then the limits to avoid collisions with the tendons are $0<\theta_{1}<3 \pi / 2$ and $-\pi / 2<\theta_{2}<\pi$. The computed workspace is shown in Figure 7 , where an instance of the five-bar linkage is also given for perspective.

\subsection{Systematic in-hand manipulation map and planning}

In this section, the prehensile in-hand manipulation capabilities of the gripper are demonstrated. First, a manipulation map is generated which relates the planar position of the center of the object and its orientation to the configuration of the five-bar linkage. Algorithm 1 describes the method of computing the mapping between the object manipulation workspace and the active joint space, which describes the possible combinations of $\theta_{1}$ and $\theta_{2}$. All the feasible 


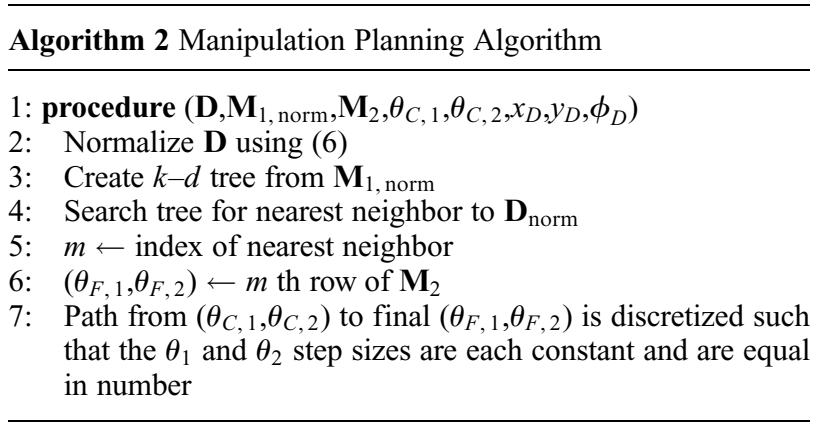

combinations of $\theta_{1}$ and $\theta_{2}$ are then swept through and the center of the object, whose coordinates are given by $x$ and $y$, is determined using the method described in Section 3.2.

In addition, the orientation of the object, denoted by $\phi$, is given by the anti-clockwise angle from the $x$-axis to the vector from $\mathbf{P}_{2}$ to $\mathbf{P}_{4}$; this is computed using the twoargument inverse tangent function so that the direction of the angle is determined. Each feasible object pose is stored in the $k$ th row of a matrix, say $\mathbf{M}_{1}$, and the corresponding joint angles make up the $k$ th row of another matrix, say $\mathbf{M}_{2}$. As the units of $\mathbf{M}_{1}$ are not homogeneous, $\mathbf{M}_{1}$ is normalized, such that

$$
\mathbf{M}_{1, \text { norm }, i}=\left[\begin{array}{c}
\frac{x_{i}-\min (x, y)}{\max (x, y)-\min (x, y)} \\
\frac{y_{i}-\min (x, y)}{\max (x, y)-\min (x, y)} \\
\frac{\phi_{i}-\min (\phi)}{\max (\phi)-\min (\phi)}
\end{array}\right]^{\mathrm{T}}
$$

where $\mathbf{M}_{1, \text { norm, } i}$ denotes the $i$ th row of the normalized $\mathbf{M}_{1}$ matrix, $\left(x_{i}, y_{i}, \phi_{i}\right)$ make up the $i$ th row of $\mathbf{M}_{1}, \min (x, y) /$ $\max (x, y)$ denotes the minimum/maximum of all $x$ and $y$ values, and $\min (\phi) / \max (\phi)$ denotes the minimum/maximum of all $\phi$ values.

Algorithm 2, the computation of manipulation planning, utilizes the above mapping to find the shortest distance in joint space to move from the current pose of the manipulator to the desired pose, $\mathbf{D}=\left(x_{D}, y_{D}, \phi_{D}\right)$. Here $\mathbf{D}$ is normalized in the same manner as $\mathbf{M}_{1 \text {, norm }}$, such that

$$
\mathbf{D}_{\text {norm }}=\left[\begin{array}{c}
\frac{x_{D}-\min (x, y)}{\max (x, y)-\min (x, y)} \\
\frac{y_{D}-\min (x, y)}{\max (x, y)-\min (x, y)} \\
\frac{\phi_{D}-\min (\phi)}{\max (\phi)-\min (\phi)}
\end{array}\right]^{\mathrm{T}}
$$

Now, in order to find the nearest neighbor in $\mathbf{M}_{1 \text {, norm }}$ to $\mathbf{D}_{\text {norm, a }} k-d$ tree is formed from $\mathbf{M}_{1 \text {, norm. This tree is }}$ formed by taking the median of the points in $\mathbf{M}_{1 \text {, norm }}$ with respect to a particular coordinate (this point is called the root), and splitting the set into two; the subset of points to the left of the root comprise the left side of the tree and those to the right comprise the right side of the tree. The median of each of these sets is found with respect to the next coordinate and the tree is formed by continuing to partition all of the points in this fashion. The $k-d$ tree is then used to perform a nearest-neighbor search, such that the point in $\mathbf{M}_{1, \text { norm }}$ that is the shortest Euclidean distance from $\mathbf{D}_{\text {norm }}$ is found. This is performed by starting at the root and moving down the tree depending on whether the coordinate of the desired point corresponding to the current partition is to the left or the right of the partition.

If a point in the tree is reached which is closest so far to the desired point, it is recorded as such. The possibility that there are points on the other side of the partition that are closer is checked by forming a sphere around the desired point with a radius equal to that of the distance between the current closest point and the desired point: if the sphere crosses the partitioning plane, there could be closer points and, therefore, the opposite branch must be checked, otherwise the opposite branch can be neglected. This algorithm continues until the nearest neighbor is found. The index, $m$, of this point is taken and the final joint coordinates, $\left(\theta_{F, 1}, \theta_{F, 2}\right)$, are given by the $m$ th row of $\mathbf{M}_{2}$, the matrix of joint angles.

The path from the current pose of the manipulator, defined by the joint angles $\left(\theta_{C, 1}, \theta_{C, 2}\right)$, to the final pose is discretized such that the $\theta_{1}$ and $\theta_{2}$ step values are constant and equal in number. Figure 8 shows the manipulation map of the gripper with three examples trajectories of the object; Figure 8(a) and (b) show the trajectories of the object from two different views and Figure 8(c) shows the corresponding joint angle profiles.

\section{Performance evaluation}

To show how the five-bar linkage of the RUTH gripper and proposed manipulation strategy impact the grasping and inhand manipulation behavior, a series of objects, including both regular objects (e.g., cylinders, squares) and daily-life objects, were used for assessment. The daily-life objects were taken from the YCB object set (Calli et al., 2015). The grasping and in-hand manipulation tasks included picking up and grasping an object from a workbench with the three grasping configurations, and manipulating an object in the air across the gripper workspace. A total of 15 objects of various sizes and shapes were used for in-hand translation and rotation tasks, and 28 objects were grasped as detailed in the YCB Gripper Assessment Benchmark (Calli et al., 2015). This section highlights the five-bar reconfigurable palm and other design features illustrated by the experiments. Videos from the experiments are included with this article as Extensions 2 and 3.

\subsection{Experimental setup}

The RUTH gripper was attached to a Universal Robot Arm (UR5) for performing grasping tests. An Arduino Nano was used to control the movement of the five-bar linkage (reconfigurable palm) and the grasping through the Dynamixel motors. Motion tracking cameras (OptiTrack 


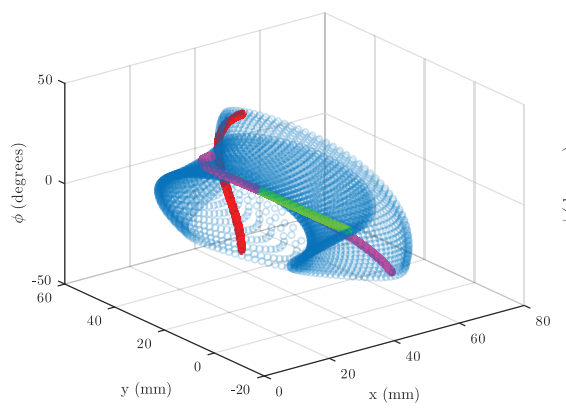

(a)

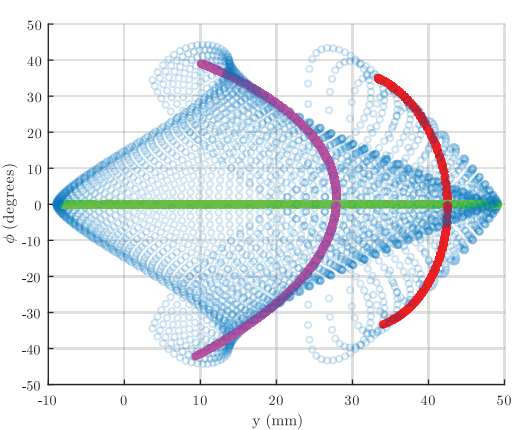

(b)

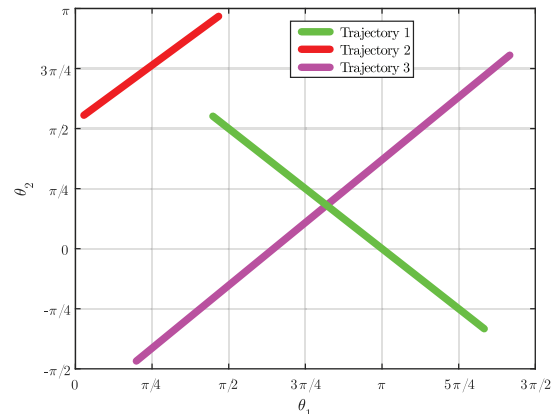

(c)

Fig. 8. The manipulation map with the three tested trajectories of the grasped object. Trajectory 1 is a pure translation, trajectory 2 is a pure rotation, and trajectory 3 is a combined translation and rotation: (a) and (b) two different views of the object trajectories across the hand workspace; (c) shows the corresponding joint angle profiles.

Flex3) were used to track the testing object trajectories, where all these objects had four tracking markers on them. Each in-hand manipulation test consisted of five repeated trials to generate reliable performance results.

\subsection{Grasping capability and workspace}

By taking advantage of the five-bar linkage palm, the RUTH gripper is able to grasp various objects in the different grasping configurations shown in Figure 6. The grasping capability of the RUTH gripper was tested by performing the YCB Gripper Assessment Benchmark (Calli et al., 2015), grasping a range set of YCB objects which include a set of spheres ranging in size from 17.4 to $145 \mathrm{~mm}$, a set of tools, flat objects, and articulated objects (see Figure 9).

The grasping tests were carried out not only to show the grasping capabilities of the gripper, but additionally to show how the reconfigurability of the gripper increases the number of objects that can be grasped. First, all of the objects were attempted to be grasped using the trigonal planar grasp posture, as shown in Figure 6(b); this grasp was tested first as its symmetrical nature allows force closure for a greater range of objects compared with the other two grasps. The grasp procedure was carried out for each object (excluding the two articulated objects) as follows. First, the hand was moved into the correct grasping position and the fingers were closed to grasp the object. Then, the gripper was raised by $30 \mathrm{~cm}$ and would remain in this position for 3 seconds. The gripper would then be rotated about the $x$ axis, an axis which is parallel to the surface of the table, and then remain in this position for 3 seconds. The grasp was then given a score between zero and four depending on the success of the test. If the initial grasp failed, or if the object was dropped during the raising motion, a score of zero would be awarded. If the object remained in the grasp after being in the raised position for 3 seconds but had visibly moved within the grasp, a score of one was awarded. If the object remained in the grasp with no visible movement up to this point, a score of two was awarded. Similarly, if

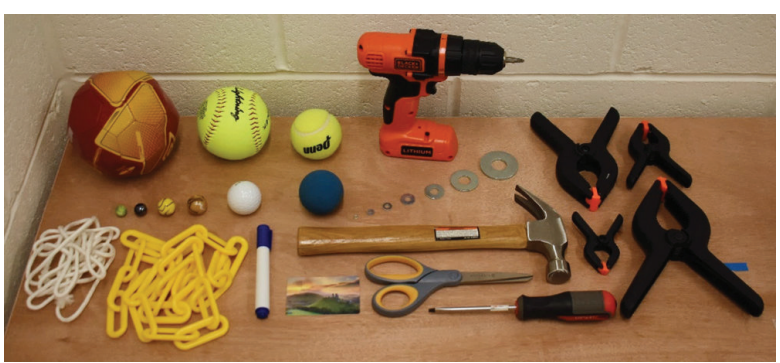

Fig. 9. The YCB object set, used entirely in the grasping capability evaluation and partially in the in-hand manipulation evaluation of the gripper.

the object was dropped after being rotated and held there for 3 seconds, no additional score was awarded. If the object had stayed in the grasp but visibly moved during this process, an additional score of one was awarded (scoring a total of three). If a secure grasp was maintained during this process, an additional score of two was awarded (scoring a total of four).

In order to test the robustness to uncertainty in the object's position, the grasping procedure was attempted for each object in four different positions. First, the object was placed onto a flat, 1-cm-thick surface which was placed on top of the table, and the grasping procedure described previously was performed. Then, the same procedure was performed, with the same initial gripper position, for three other object positions, corresponding to disturbance along the $x$-, $y$-, and $z$-axes. It should be noted that the $z$-axis disturbance is measured for the round objects and the tools, but not the flat objects. The $x$ - and $y$-axes correspond to the orthogonal axes which form the plane corresponding to the surface of the table, the $z$-axis is orthogonal to the surface of the table, and the origin is defined by the initial object position. The disturbance along the $x$ - and $y$-axes is performed by moving the object $1 \mathrm{~cm}$ along each of the axes, respectively. The disturbance along the $z$-axis is performed by removing the $1-\mathrm{cm}$-thick surface and placing the object 


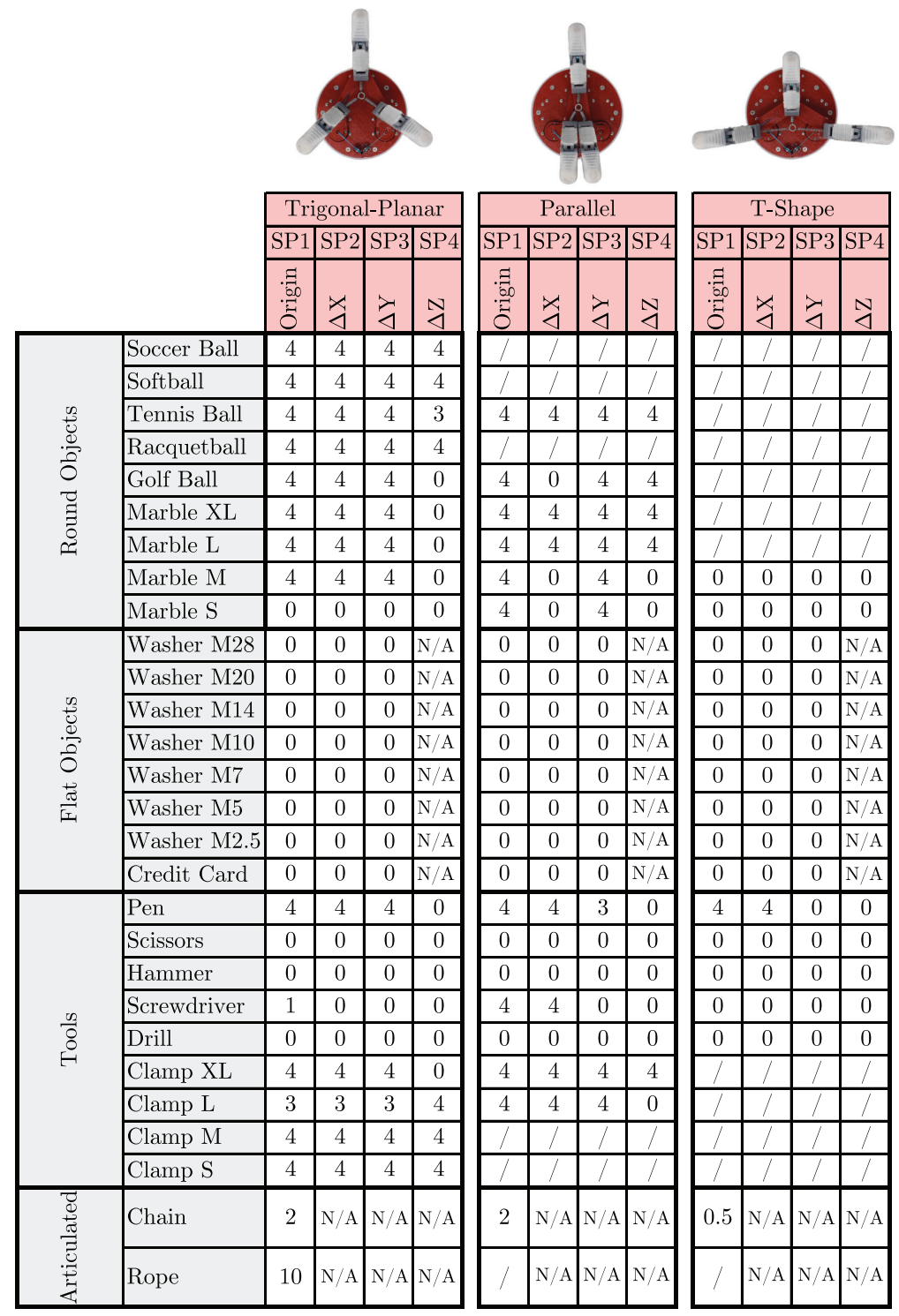

Fig. 10. Experimental results of the YCB Grasping Benchmark. All of the objects were attempted to be grasped using the trigonal planar grasp posture first, with failed objects repeated with parallel and T-shape grasp configurations in sequence. Repeats are stopped (“/”) once the full score for an object can be achieved.

on the table directly below the initial object position. The grasping procedure was carried out, and a score was given, for each of the objects in each of the four positions.

One of the advantages of the RUTH hand is that its reconfigurability allows different grasp postures to be achieved, which increases the potential number of objects that are able to be grasped. After carrying out the grasping procedure using the trigonal planar grasp and collecting the scores for each object in each position, there are some objects which received a maximum score, and some that did not. The grasping procedure was performed again on those objects that did not achieve a maximum score, but this time using the parallel grasp, as shown in Figure 6(a). Similarly, after grasping with the parallel grasp, the grasping procedure was performed again on those objects that still had not achieved a combined maximum score, this time using the T-shape grasp, as shown in Figure 6(c). The results are given in Figure 10.

The two articulated objects follow a different grasping procedure and scoring method. The object is grasped, raised by $30 \mathrm{~cm}$, and held there for 3 seconds. If the object remains in the grasp with no part of it touching the ground, 0.5 points is awarded. This is repeated 20 times, giving a total possible score of 10 . Similarly to the rigid object, if the articulated object did not receive a perfect score with the trigonal planar grasp, then it was repeated with the planar a grasp, and then the T-shape grasp. The results for the articulated objects are given at the bottom of Figure 10.

In addition to the YCB Grasping Benchmark tests, the grasping workspace was measured by grasping the $50 \mathrm{~mm}$ 
Table 1. Simulated translation and rotation quantities of the desired three trajectories for the in-hand manipulation evaluation

\begin{tabular}{lll}
\hline & \multicolumn{2}{l}{ Simulated trajectory motion } \\
\cline { 2 - 3 } Trajectory & Translation $(\mathrm{mm})$ & Rotation \\
\hline T1 & 57.2 & $0^{\circ}$ \\
T2 & 0.7 & $68.2^{\circ}$ \\
T3 & 54.9 & $81.2^{\circ}$ \\
\hline
\end{tabular}

cylindrical object at the boundary case and comparing it to the simulated workspace shown in Figure 12. The blue dots are the feasible manipulation workspace of the RUTH gripper in the $x-y$ plane produced by MATLAB. The red dots correspond to the motion tracking data of the grasped object center positions. The experimental data verified the gripper grasping workspace with a little deviation due to the underactuated finger design and inconsistent grasping force.

\subsection{Systematic prehensile in-hand manipulation}

As proposed in Section 3.3, the prehensile in-hand manipulation map of the gripper was generated in terms of $x, y$, and $\phi$ using Algorithm 1. The grasped object can be moved into a desired pose by using the map to identify the $\theta_{1}$ and $\theta_{2}$ values as described in Algorithm 2. Three characteristic trajectories of the object were chosen for the tests. During the tests, the gripper was given the $\theta_{1}$ and $\theta_{2}$ values produced by the map by inputting the target $x, y$, and $\phi$. The change of $\theta_{1}$ and $\theta_{2}$ during the manipulation are linear as shown in Figure 8(c).

To evaluate the manipulation capability of the hand, multiple objects were tested along each of the three trajectories. For the regular objects, six cylindrical objects varying from 30 to $90 \mathrm{~mm}$ along with a cube, a hexagonal prism, and a triangular prism all of size $50 \mathrm{~mm}$ were used, as well as six objects from the YCB object set (see Figure 11). The three testing trajectories were chosen such that the first trajectory resulted in a pure translation of the object $(57.2 \mathrm{~mm})$, the second trajectory resulted in a pure rotation of the object $\left(68.2^{\circ}\right)$, and the third trajectory resulted in a combined translation and rotation of the object $\left(54.9 \mathrm{~mm}, 81.2^{\circ}\right)$. A summary of these values is given in Table 1. These trajectories can be seen visually in Figure 8. The positions of each of the manipulated objects were tracked using reflective markers placed on each of the objects. The position of the gripper base was also tracked, enabling the mapping of the simulated object trajectory to the tracking coordinate system. The difference between the simulated object trajectory and each experimental object trajectory were measured and reported. The translation error along each axis $(x / y / z)$, where $z$ is the vertical axis and $x$ and $y$ are the planar axes, and the rotation error around the $z$-axis and from the $z$-axis (reported as "tilt"), are reported. A diagram demonstrating each of these errors

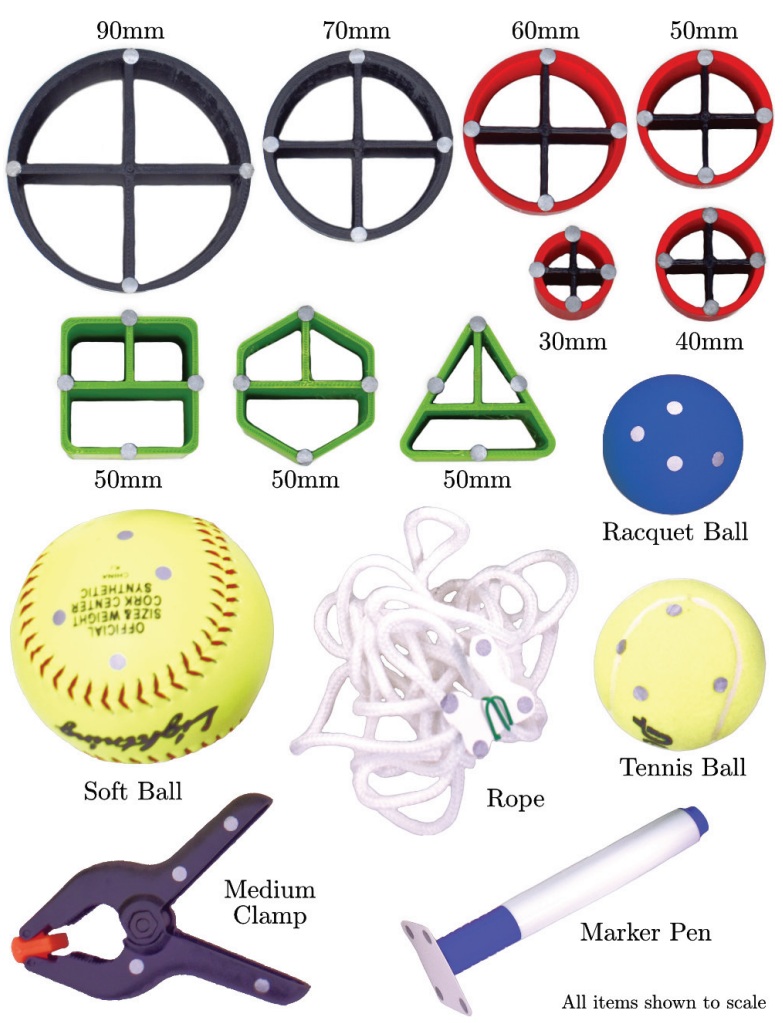

Fig. 11. Regular objects and a subset of the YCB objects used in the in-hand manipulation evaluation of the gripper, with tracking marker positions shown.

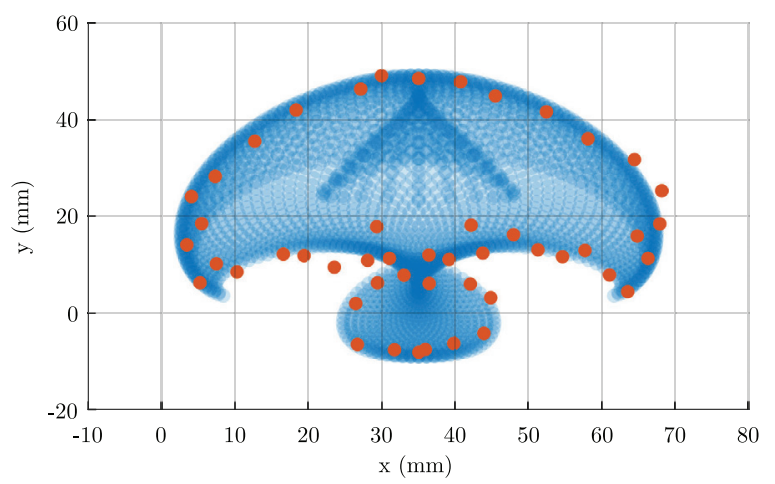

Fig. 12. Experimental object positions at the RUTH gripper's configuration boundary (red). Simulation workspace of the RUTH gripper (blue).

can be seen in Figure 13. These errors are reported as both an average error across the entire trajectory, as well as just at the desired end point of the trajectory. Each trajectory was repeated five times for each object, with the average then taken to improve accuracy. Figure 14 shows an example experimental object's trajectories compared with the simulation trajectories. The translation error results of the manipulation test can be seen in Figures 15 and 16, whereas the rotation error results can be seen in Figures 17 and 18 . 

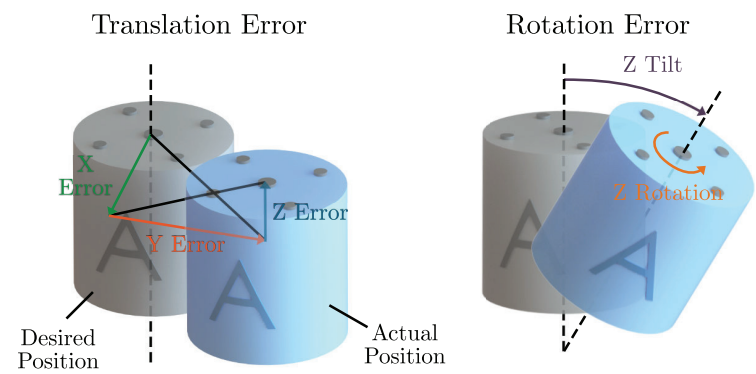

Fig. 13. Diagram demonstrating how the translation (left) and rotation (right) positioning errors relative to the desired object location are reported.

\section{Discussion}

\subsection{Grasping performance}

The performance of the RUTH gripper in the YCB Manipulation Benchmark demonstrates how the different grasp configurations are advantageous, as they can achieve a successful grasp where other configurations might not succeed. In the trigonal planar grasp, we see a complete success (that is, full marks achieved in grasping at the object origin, as well as in the $x, y$, and $z$ offsets) in the larger spherical objects, as well as the smaller clamps and rope. The smaller spherical objects fail with the $z$ offset, which can be explained by the gripper no longer grasping below the center of the sphere, causing the objects to fall out of the gripper. For the smallest spherical object, it was not possible to grasp below the center point in any of the object positions. In the case of the articulated objects, the rope shows complete success as it is quite light, and holds its form when grasped, unlike the chain which is significantly heavier and requires a more encapsulating grasp, explaining its limited success. For the tools, we saw success with the majority of the clamps, as well as success with the pen, which showed a similar issue to the spherical objects in the $z$ offset. The drill and hammer were shown to be too heavy for the RUTH gripper, with the object's weight causing the object to slide out of the grasp. Weight also showed to be an issue for the screwdriver, where the balance of the weight caused the object to rotate out of the grasp. The scissors were not too heavy, however were too flat to be grasped by the gripper. None of the flat objects were successful, and we believe this can be attributed to the underactuated finger design, providing limited control over balancing the bending of the finger phalanges, as well as the fingers lacking optimization for flat objects, such as not including a fingernail. In all configurations, the gripper was unable to lift the flat objects off of the table, and any recorded movement typically resulted in the object being pushed out of the grasping area. For the purely trigonal planar grasp, a score of 193/404 was achieved, giving a successful percentage of $48 \%$. Removing flat objects from scoring, this scoring is $193 / 260$, with a grasp percentage of $74 \%$.

Objects that did not show complete success were repeated in the parallel grasp, and then again in the T-shape grasp if a complete success had still not been achieved. The parallel grasp showed success in areas where the trigonal planar had failed, such as maintaining a grasp with the $\mathrm{Z}$ offset on the medium (tennis ball) and smaller spherical objects. Some success was also shown for the screwdriver and the larger clamps, possibly due to the slightly higher grasp force achievable in the parallel grasp. No additional successes that had not previously been achieved were measured with the T-shape grasp, however this was expected as the main advantage of the T-shape grasp is the increased grasping size, and the largest objects of the YCB set had already shown complete success in the trigonal planar grasp. With the additional scores from the parallel grasp, the total score was increased to $228 / 404$, a percentage of $56 \%$. Removing the flat objects category this scoring is $228 / 260$, a percentage of $88 \%$.

From these results we can see the grasp configurations do indeed provide an advantage in grasping, allowing an increased number of objects to be successfully grasped. We agree that the T-shaped configuration does not add much to the grasping capability. We obtained this configuration to evaluate the gripper in extreme position; it might be useful for some specially designed objects. The five-bar linkage design can achieve different grasping configurations,

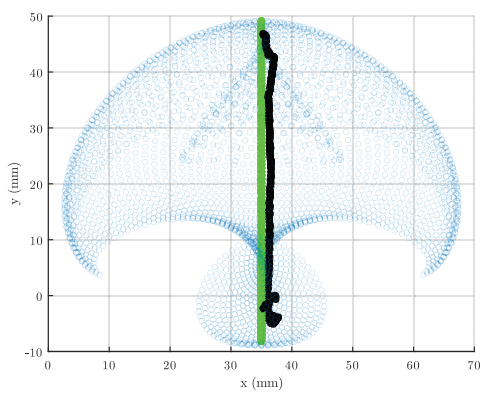

(a)

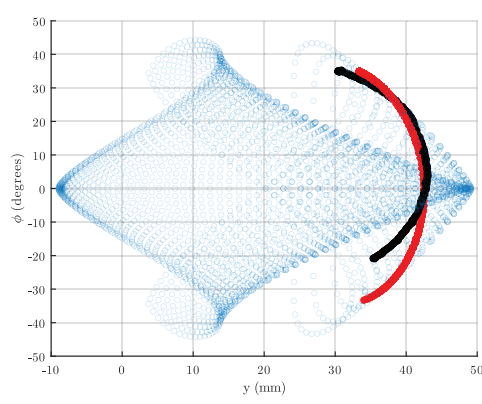

(b)

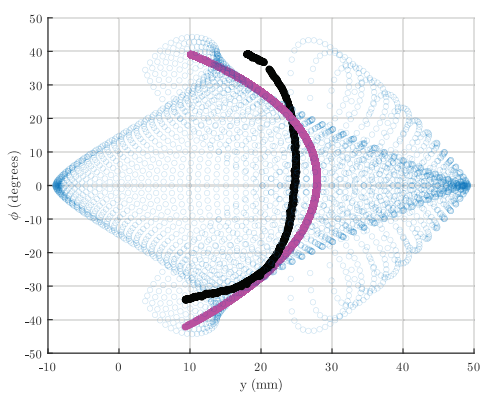

(c)

Fig. 14. Experimental motion tracking object trajectories (black) overlaid on simulated trajectories: (a) pure translation, (b) pure rotation, and (c) combined translation and rotation. 


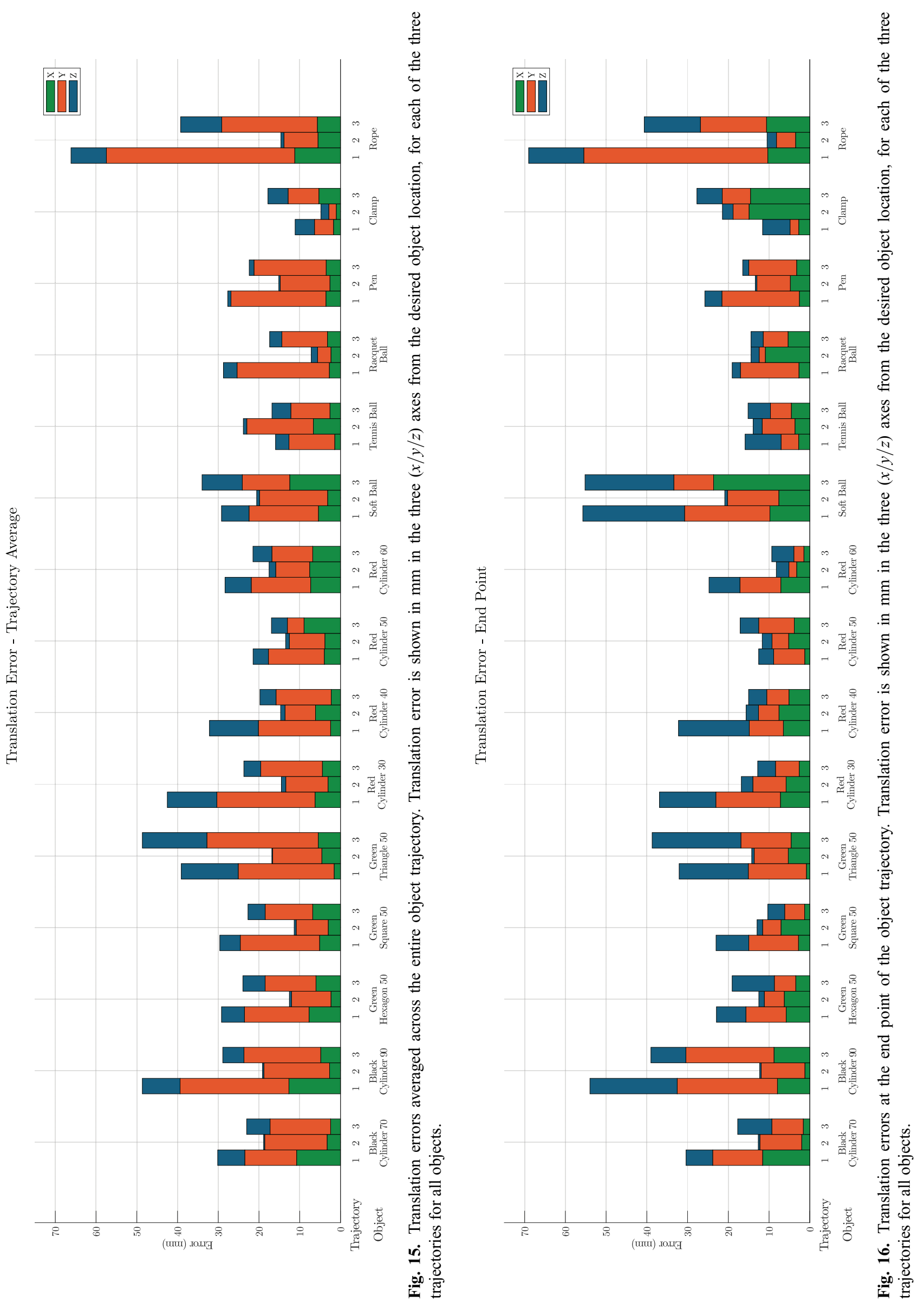




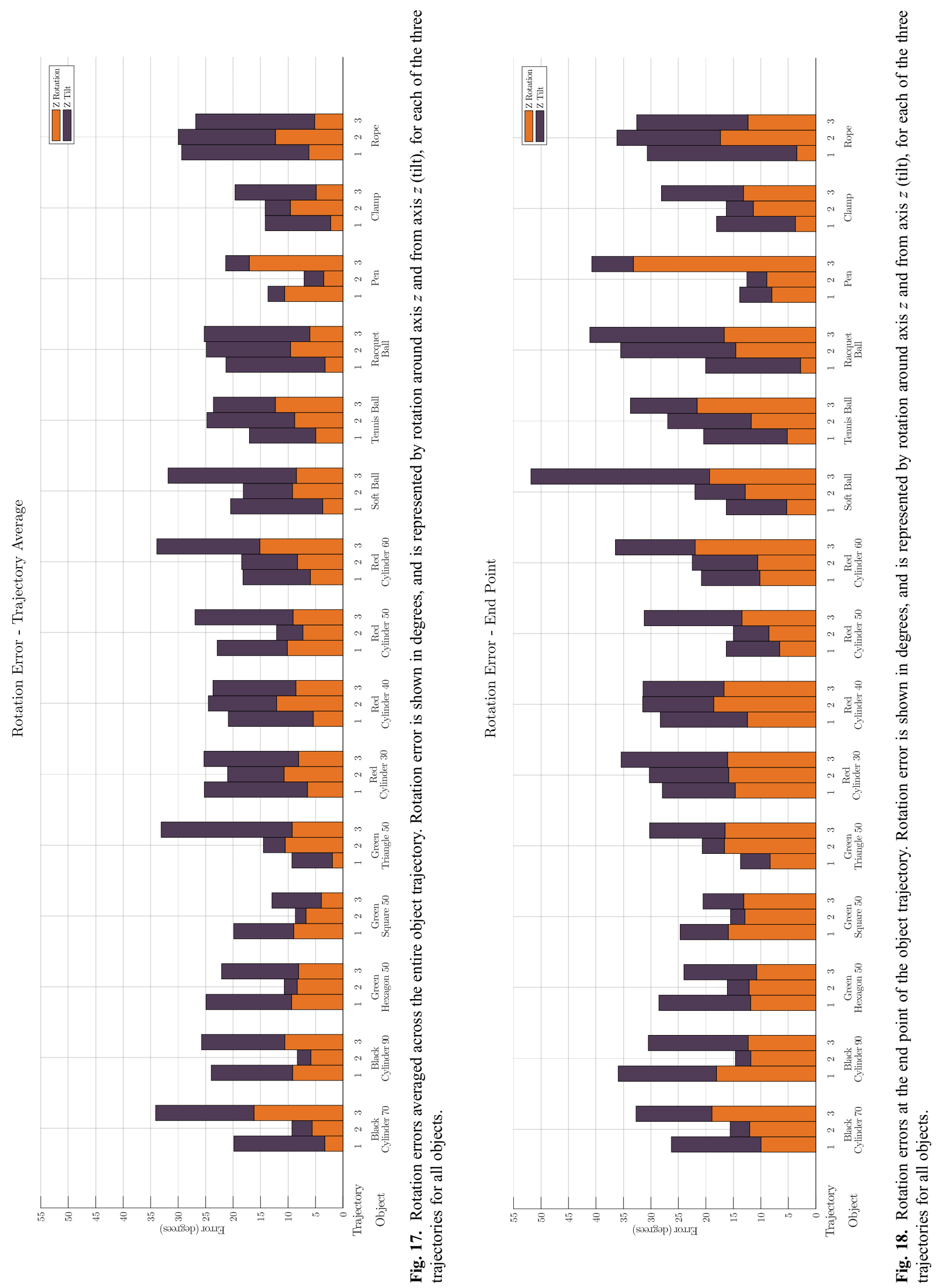


similar to other reconfiguration hands, such as the BarrettHand. Moreover, the 5-bar linkage can also achieve systematic in-hand manipulation. Limitations of the gripper were highlighted, such as the fingertip design and phalange control for flat objects, as well as the force transmission through the tendon routing system restricting the maximum force output making grasping heavy objects difficult. Objects outside of these two categories, however, showed consistent success.

\subsection{In-hand manipulation performance}

The overall performance of the RUTH gripper in manipulating an object is predictable with some deviations, especially for non-cylindrical objects. Across all objects, we observe a minimal error in the $x$-axis $(<10 \mathrm{~mm})$, a consistent error of $5-15 \mathrm{~mm}$ for the $y$-axis, and either almost no error $(<5$ $\mathrm{mm}$ ) or a larger $10-20 \mathrm{~mm}$ error in the $z$-axis. For noncylindrical objects, the distances from each finger contact point to the center of the object vary during the manipulation, which is a challenge for an underactuated gripper. The translation errors may occur, at least partly, owing to the gripper grasp-pushing the manipulated object away from the center of the gripper. This is reflected by the greater error in the translation along the $y$-axis, as opposed to the $x$-axis, for most of the trials, as the fingers attached at $P_{2}$ and $P_{4}$ tend to push the object more toward the finger attached at $P_{3}$. This also explains the consistency across the $y$-axis errors, with the majority of objects showing an error of $\sim 10 \mathrm{~mm}$. This may easily affect both the average mean and final position of the object and contribute to the translation error. For the proposed design, in order to have a constant tendon routing design for the five-bar linkage (the reconfigurable palm) the tendon routing, shown in Figure 2, may reduce the force transmission efficiency significantly given the small-radii pulleys that are used, resulting in a limited ability to push the object towards the center of the gripper.

As discussed previously, the translation errors tended to be relatively small for the cylindrical objects, with the exception being the largest of them: the black cylinder with a $90 \mathrm{~mm}$ diameter. However, for the rotational errors the opposite is true, with the smaller cylinders performing worse than the larger cylinders. This is likely to be because for the larger cylinders, there were not only contacts at the fingertips but also at other points on the fingers, which may have helped keep these objects more upright during the manipulation. This was also the case for the pen, the end of which was in contact with the base of the gripper throughout the manipulation, and, as a result, both the mean and final tilting error is quite small.

Another contributing factor to the translation errors is the changing contact points during the prehensile in-hand manipulation. In the simulation, it is assumed that the object is always being grasped at the center of the hand and the grasping configuration of each finger is identical. However, in reality, there are some aspects that make these assumptions not totally valid. First, the three three-phalanx fingers are actuated by only one motor via tendons using torque control. To maintain a constant grasp during a manipulation, the torque control of the tendons will tighten or release the tendons to achieve a consistent finger force on the object. With the same change in the length of the tendons, each finger configuration (the angles between phalanges) may still vary due to other factors, such as the contact force, manufacturing errors, and structural friction. With this uncertainty, each of the three fingers may end up with a slightly different bending height which will cause object translation and rotation errors. In addition, the manipulation has not been tested in the "upside down" configuration, where the performance is expected to be worse. Taking into account these considerations into the simulation is still an open research question. In the case of loosely held objects, such as the rope, this change in contact points may also explain the slippage of the object, resulting in an increased $z$-axis error. The same is true for heavy objects (soft ball, black cylinder 90), and objects that do not adapt well with a change in finger configuration (green triangle 50). This is confirmed by the same objects showing little to no $z$-axis error in the second trajectory, where the finger configuration is constant across the entire trajectory.

To maintain the grasping capability as the five-bar configuration changes, the fingers actuation motion should be towards the center of the triangle formed by the three finger base positions proposed in Section 2. To achieve this, the orientation of each finger was controlled by a high-stiffness spring attached at the base of each finger to a central ring. In some cases, when the gripper grasped an object tightly, the central ring may struggle to pull all the fingers towards the center of the triangle which may produce the position error adding to the end position.

In terms of the trajectories, it is clear from the results that the displacement error tends to be lower for Trajectory 2. This is likely because the length of the total trajectory is smaller than that of the other two, and additionally the end point position of the object should be equal to its start position. Similarly, the smaller rotational errors for this trajectory can be accounted for by its shorter total length, as well as the previously mentioned lack of gripper configuration change across the trajectory. The rotational errors for Trajectory 1, the straight translation trajectory, are reasonably small, especially the rotational error about the $z$-axis. This is likely due to the fact that the aim for this trajectory does not include a rotation around the $z$-axis, and so the fingers sliding over the surface of the object produced little unwanted rotation. It is expected that Trajectory 3 suffered the highest errors, in both translation and rotation, as it was both the longest trajectory but also included a rotational aspect as well.

\section{Conclusion}

In this article, the design, construction, and evaluation of a reconfigurable underactuated constant-tendon hand (the 
RUTH gripper) has been presented, which decouples manipulation and grasping to facilitate the control and implementation of prehensile in-hand manipulation. Through the use of a five-bar linkage as the palm of the gripper, the fingers are capable of repositioning to allow not just different grasp types, but object-invariant in-hand manipulation. The design of the reconfigurable palm has been explored, as has the method of achieving underactuated constant-tendon routing despite the ability of each finger to change its proximal joint position. An algorithm to compute the feasible manipulation map using distancebased kinematics has been proposed, as has an algorithm for manipulation planning and control. The hand has been experimentally evaluated in both grasping and in-hand manipulation capabilities. A wide range of objects have been tested for the grasping capability under different grasping configurations. Nine sample objects of different size and shape and six every-day objects were manipulated in three trajectories using the algorithms proposed. From the results, we see that with the proposed mechanicalintelligence design principle, the gripper can achieve precise systematic in-hand manipulation regardless of the particularities of the object with a simple control scheme.

For future work, force analysis can be performed to calculate the required torque for the constant-tendon routing, and this routing can be optimized to improve the force transmission efficiency. Moreover, the introduced graspingmanipulation decoupling approach can be explored for sixdimensional in-hand manipulation through a novel design of the base linkages or an improved underactuated finger design. Overall, the work in this article opens up a new idea on co-designing robot hand and control for dexterous in-hand manipulations.

\section{Funding}

The authors disclosed receipt of the following financial support for research, authorship and/or publication of this article: This work was supported in part by the Engineering and Physical Sciences Research Council (grant number EP/R020833/1) and an Amazon Research Award.

\section{References}

Aukes DM, Heyneman B, Ulmen J, et al. (2014) Design and testing of a selectively compliant underactuated hand. The International Journal of Robotics Research 33(5): 721-735.

Bai G and Rojas N (2018) Self-adaptive monolithic anthropomorphic finger with teeth-guided compliant cross-four-bar joints for underactuated hands. In: International Conference on Humanoid Robots. IEEE, pp. 145-152.

Baron N, Philippides A and Rojas N (2019) A novel kinematically redundant planar parallel robot manipulator with full rotatability. Journal of Mechanisms and Robotics 11(1): 011008.

Bicchi A (2000) Hands for dexterous manipulation and robust grasping: A difficult road toward simplicity. IEEE Transactions on Robotics and Automation 16(6): 652-662.

Billard A and Kragic D (2019) Trends and challenges in robot manipulation. Science 364(6446): eaat8414.
Bouman A, Nadan P, Anderson M, et al. (2020) Design and autonomous stabilization of a ballistically-launched multirotor. In: 2020 IEEE International Conference on Robotics and Automation (ICRA). IEEE, pp. 8511-8517.

Calli B, Singh A, Walsman A, Srinivasa S, Abbeel P and Dollar AM (2015) The YCB object and model set: Towards common benchmarks for manipulation research. In: International Conference on Advanced Robotics. IEEE, pp. 510-517.

Catalano MG, Grioli G, Farnioli E, Serio A, Piazza C and Bicchi A (2014) Adaptive synergies for the design and control of the Pisa/IIT SoftHand. The International Journal of Robotics Research 33(5): 768-782.

Chavan-Dafie N, Lee K and Rodriguez A (2018) Pneumatic shape-shifting fingers to reorient and grasp. In: International Conference on Automation Science and Engineering. IEEE, pp. 988-993.

Ciocarlie M, Hicks FM, Holmberg R, et al. (2014) The Velo Gripper: A versatile single-actuator design for enveloping, parallel and fingertip grasps. The International Journal of Robotics Research 33(5): 753-767.

Cui X, Sun J, Zhang XS, Xu SJ and Dai JS (2018) A metamorphic hand with coplanar reconfiguration. In: International Conference on Reconfigurable Mechanisms and Robots. IEEE, pp. 1-7.

Dai JS, Wang D and Cui L (2009) Orientation and workspace analysis of the multifingered metamorphic hand - Metahand. Transactions on Robotics 25(4): 942-947.

Della Santina C, Piazza C, Grioli G, Catalano MG and Bicchi A (2018) Toward dexterous manipulation with augmented adaptive synergies: The Pisa/IIT SoftHand 2. IEEE Transactions on Robotics 34(5): 1141-1156.

Dollar A (2006) Design Principles for Robust Grasping in Unstructured Environments. PhD Thesis, Harvard University.

Dollar AM and Howe RD (2010) The highly adaptive SDM hand: Design and performance evaluation. The International Journal of Robotics Research 29(5): 585-597.

Dollar AM and Howe RD (2011) Joint coupling design of underactuated hands for unstructured environments. The International Journal of Robotics Research 30(9): 1157-1169.

Eppner C, Deimel R, Alvarez-Ruiz J, Maertens M and Brock O (2015) Exploitation of environmental constraints in human and robotic grasping. The International Journal of Robotics Research 34(7): 1021-1038.

Grebenstein M, Albu-Schäffer A, Bahls T, et al. (2011) The DLR hand arm system. In: 2011 IEEE International Conference on Robotics and Automation. IEEE, pp. 3175-3182.

Hang K, Bircher WG, Morgan AS and Dollar AM (2020) Handobject configuration estimation using particle filters for dexterous in-hand manipulation. The International Journal of Robotics Research 39(14): 1760-1774.

Higashimori M, Jeong H, Ishii I, Kaneko M, Namiki A and Ishikawa M (2005) A new four-fingered robot hand with dual turning mechanism. In: International Conference on Robotics and Automation. IEEE, pp. 2679-2684.

Hirose S and Umetani Y (1978) The development of soft gripper for the versatile robot hand. Mechanism and Machine Theory 13(3): 351-359.

Jacobsen S, Iversen E, Knutti D, Johnson R and Biggers K (1986) Design of the Utah/MIT dextrous hand. In: International Conference on Robotics and Automation, Vol. 3. IEEE, pp. 1520-1532.

Kawasaki H, Komatsu T and Uchiyama K (2002) Dexterous anthropomorphic robot hand with distributed tactile sensor: Gifu Hand II. Transactions on Mechatronics 7(3): 296-303. 
Lee K, Wang Y and Zheng C (2020) Twister hand: Underactuated robotic gripper inspired by origami twisted tower. IEEE Transactions on Robotics 36(2): 488-500.

Liu H, Zhang Z, Zhu X and Xu K (2018) A single-actuator gripper with a working mode switching mechanism for grasping and rolling manipulation. In: 2018 IEEE/ASME International Conference on Advanced Intelligent Mechatronics (AIM). IEEE, pp. 359-364.

Lu Q, Clark AB, Shen M and Rojas N (2020) An origami-inspired variable friction surface for increasing the dexterity of robotic grippers. IEEE Robotics and Automation Letters 5(2): 2538-2545.

Lu Q, Liang H, Thrishantha N and Rojas N (2020) Precise inhand manipulation of delicate objects using soft fingertips with adaptive sensing and active deformation. In: International Conference on Soft Robotics.

Lu Q and Rojas N (2019) On soft fingertips for in-hand manipulation: Modeling and implications for robot hand design. Robotics and Automation Letters 4(3): 2471-2478.

Ma RR, Odhner LU and Dollar AM (2013) A modular, open-source 3D printed underactuated hand. In: 2013 IEEE International Conference on Robotics and Automation. IEEE, pp. 2737-2743.

Ma RR, Rojas N and Dollar AM (2016) Spherical hands: Toward underactuated, in-hand manipulation invariant to object size and grasp location. Journal of Mechanisms and Robotics 8(6): 061021.

Morino K, Kikuchi S, Chikagawa S, Izumi M and Watanabe T (2020) Sheet-based gripper featuring passive pull-in functionality for bin picking and for picking up thin flexible objects. IEEE Robotics and Automation Letters 5(2): 2007-2014.

Nagabandi A, Konolige K, Levine S and Kumar V (2020) Deep dynamics models for learning dexterous manipulation. In: Conference on Robot Learning. PMLR.org, pp. 1101-1112.

Odhner LU, Jentoft LP, Claffee MR, et al. (2014) A compliant, underactuated hand for robust manipulation. The International Journal of Robotics Research 33(5): 736-752.

Okamura AM, Smaby N and Cutkosky MR (2000) An overview of dexterous manipulation. In: Proceedings 2000 ICRA. Millennium Conference. IEEE International Conference on Robotics and Automation, Vol. 1. IEEE, pp. 255-262.

Open AI, Akkaya I, Andrychowicz M, et al. (2019) Solving Rubik's Cube with a robot hand. arXiv preprint arXiv:1910.07113.

Open AI, Andrychowicz OM, Baker B, et al. (2020) Learning dexterous in-hand manipulation. The International Journal of Robotics Research 39(1): 3-20.

Piazza C, Grioli G, Catalano M and Bicchi A (2019) A century of robotic hands. Annual Review of Control, Robotics, and Autonomous Systems 2: 1-32.

Righthand Robotics (2019) ReFlex robotic gripper. https:// www.labs.righthandrobotics.com/reflexhand (accessed 19 September 2021).

Robotiq (2016) 3-finger adaptive robot gripper. https://robotiq.com/products/3-finger-adaptive-robot-gripper (accessed 19 September 2021).

Rojas N (2012) Distance-Based Formulations for the Position Analysis Of Kinematic Chains. PhD Thesis, Universitat Politècnica de Catalunya, Barcelona.

Rojas N, Ma RR and Dollar AM (2016) The GR2 gripper: An underactuated hand for open-loop in-hand planar manipulation. Transactions on Robotics 32(3): 763-770.

Schmitz A, Pattacini U, Nori F, Natale L, Metta G and Sandini G (2010) Design, realization and sensorization of the dexterous icub hand. In: International Conference on Humanoid Robots. IEEE, pp. 186-191.

Shadow (2019) Shadow Dexterous Hand. https://www.shadowrobot.com/dexterous-hand-series/ (accessed 19 September 2021).
Spiliotopoulos J, Michalos G and Makris S (2018) A reconfigurable gripper for dexterous manipulation in flexible assembly. Inventions 3(1): 4.

Stuart H, Wang S, Khatib O and Cutkosky MR (2017) The Ocean One Hands: An adaptive design for robust marine manipulation. The International Journal of Robotics Research 36(2): 150-166.

Teeple CB, Koutros TN, Graule MA and Wood RJ (2020) Multisegment soft robotic fingers enable robust precision grasping. The International Journal of Robotics Research 39(14): $1647-1667$.

Tincani V, Catalano MG, Farnioli E, et al. (2012) Velvet Fingers: A dexterous gripper with active surfaces. In: 2012 IEEE/RSJ International Conference on Intelligent Robots and Systems. IEEE, pp. 1257-1263.

Townsend W (2000) The BarrettHand grasper - programmably flexible part handling and assembly. Industrial Robot: An International Journal 27(3): 181-188.

Trinkle JC and Hunter JJ (1991) A framework for planning dexterous manipulation. In: Proceedings 1991 IEEE International Conference on Robotics and Automation. IEEE, pp. 1245-1251.

Walkler R (2004) Developments in dextrous hands for advanced robotic applications. In: World Automation Congress, pp. 123128.

Wang J, Lu Q, Clark A and Rojas N (2020) A passively complaint idler mechanism for underactuated dexterous grippers with dynamic tendon routing. In: Mohammad A, Dong X and Russo M (eds) Towards Autonomous Robotic Systems (TAROS 2020) (Lecture Notes in Computer Science, Vol. 12228). Cham: Springer.

Ward-Cherrier B, Rojas N and Lepora NF (2017) Model-free precise in-hand manipulation with a 3D-printed tactile gripper. IEEE Robotics and Automation Letters 2(4): 2056-2063.

Wei G, Dai JS, Wang S and Luo H (2011) Kinematic analysis and prototype of a metamorphic anthropomorphic hand with a reconfigurable palm. International Journal of Humanoid Robotics 8(03): 459-479.

Yuan S, Epps AD, Nowak JB and Salisbury JK (2020) Design of a roller-based dexterous hand for object grasping and withinhand manipulation. In: 2020 IEEE International Conference on Robotics and Automation (ICRA). IEEE, pp. 8870-8876.

\section{Appendix. Index to multimedia extensions}

Archives of IJRR multimedia extensions published prior to 2014 can be found at http://www.ijrr.org, after 2014 all videos are available on the IJRR YouTube channel at http:// www.youtube.com/user/ijrrmultimedia

\section{Table of Multimedia Extensions}

\begin{tabular}{llll}
\hline Extension & Media type & Description \\
\hline 1: & $\begin{array}{l}\text { The summary video of the } \\
\text { paper. }\end{array}$ & Video & Summary \\
2: $\begin{array}{l}\text { The grasping capability } \\
\text { video of the RUTH Gripper. }\end{array}$ & Video & Experiment \\
3: $\begin{array}{l}\text { The in-hand manipulation } \\
\text { capability video of the RUTH }\end{array}$ & Video & Experiment \\
& & \\
\hline
\end{tabular}

